\title{
Phenotype-Haplotype Correlation of IRF5 in Systemic Sclerosis: Role of 2 Haplotypes in Disease Severity
}

\author{
PHILIPPE DIEUDE, KAREN DAWIDOWICZ, MICKAËL GUEDJ, YONA LEGRAIN, JULIEN WIPFF, \\ ERIC HACHULLA, ELISABETH DIOT, JEAN SIBILIA, LUC MOUTHON, JEAN CABANE, ZAHIR AMOURA, \\ JEAN-LUC CRAKOWSKI, PATRICK CARPENTIER, JEROME AVOUAC, OLIVIER MEYER, ANDRE KAHAN, \\ CATHERINE BOILEAU, and YANNICK ALLANORE
}

\begin{abstract}
Objective. Identification of an association between IRF5 rs2004640 and systemic sclerosis (SSc) has highlighted a key role for type 1 interferon (IFN). Additional functional IRF5 variants have been identified as autoimmune susceptibility factors. Our aim was to investigate whether IRF5 haplotypes confer susceptibility to SSc, and to perform genotype haplotype-phenotype correlation analyses.

Methods. We genotyped IRF5 rs377385, rs2004640, and rs10954213 in 1623 individuals of French European Caucasian origin. SSc patient subphenotypes were analyzed according to cutaneous subsets and for SSc-related pulmonary fibrosis.

Results. Case-control studies of single markers revealed an association between IRF5 rs 3757385 , rs2004640, and rs10954213 variants and SSc. We identified an IRF5 risk haplotype " $\mathrm{R}$ " ( $\mathrm{p}_{\mathrm{adj}}=$ 0.024 , OR $1.23,95 \%$ CI 1.07-1.40) and a mirrored protective haplotype " $\mathrm{P}$ " $\left(\mathrm{p}_{\mathrm{adj}}=8.8 \times 10^{-3}\right.$, OR $0.78,95 \%$ CI $0.68-0.90$ ) for SSc susceptibility. Genotype-phenotype correlation analyses failed to detect any association with a single marker. By contrast, phenotype-haplotype correlation analysis was able to detect intra-cohort association and to discriminate SSc patients with from those without the following clinical traits: "R" and/or "P" haplotypes identified diffuse cutaneous SSc ( $\mathrm{p}=0.0081)$ and fibrosing alveolitis $(\mathrm{p}=0.018)$.

Conclusion. IRF5 haplotypes are more informative than single markers, suggesting that they could be helpful for risk stratification of SSc patients. Our study provides further evidence of a key role of IRF5 in SSc severity. (First Release March 15 2010; J Rheumatol 2010;37:987-92; doi:10.3899/ jrheum.091163)
\end{abstract}

Key Indexing Terms:

IRF5 INTERFERON HAPLOTYPE SYSTEMIC SCLEROSIS

From the Service de Rhumatologie, Hôpital Bichat Claude Bernard, APHP, Université Diderot-Paris 7, Paris; Laboratoire Statistique et Génome, UMR CNRS-8071 / INRA-1152, Université d'Evry Val d'Essonne; INSERM U781, Université Paris Descartes, Hôpital Necker, Paris; Rhumatologie A, Hôpital Cochin, APHP, Université Paris Descartes, Paris; Médecine Interne, Université Lille II, Lille; INSERM EMI-U 00-10, CHU Bretonneau, Tours; Service de Rhumatologie, Hôpital Hautepierre, Université Louis Pasteur, Strasbourg; Médecine Interne, Hôpital Cochin, APHP, Paris Descartes Université, Paris; Service de Médecine Interne, Hôpital Saint-Antoine, APHP, Université Pierre et Marie Curie, Paris; Service de Médecine Interne 2, Hôpital

Pitié-Salpêtrière, AP-HP, Université Paris 6, Paris; Laboratoire de Pharmacologie, INSERM ESPRI, HP2 EA 3745, Faculté de Médecine de Grenoble, Grenoble; Clinique Universitaire De Médecine Vasculaire, Pôle Pluridisciplinaire de Médecine, Centre Hospitalier Universitaire, Grenoble; and Laboratoire de Biochimie Hormonale et Génétique, Hôpital Ambroise Paré, AP-HP, Université Versailles Saint Quentin Yvelines, Boulogne, France.

Supported by Association des Sclérodermiques de France, Institut national de la santé et de la recherche médicale (INSERM), Agence Nationale pour la Recherche (ANR; no. R07094KS), and Groupe Français de Recherche sur la Sclérodermie.

P. Dieudé, MD, PhD; K. Dawidowicz, MD, Service de Rhumatologie, Hôpital Bichat Claude Bernard, APHP; M. Guedj, PhD, Laboratoire Statistique et Génome, UMR CNRS-8071 / INRA-1152, Université d'Evry Val d'Essonne; Y. Legrain, PhD, INSERM U781, Université Paris Descartes, Hôpital Necker; J. Wipff, MD, PhD, INSERM U781, Université Paris Descartes, Hôpital Necker, Rhumatologie A, Hôpital Cochin,
APHP; E. Hachulla, MD, PhD, Professor, Médecine Interne, Université Lille II; E. Diot, MD, PhD, INSERM EMI-U 00-10, CHU Bretonneau; J. Sibilia, MD, PhD, Professor, Université Louis Pasteur, Service de Rhumatologie, Hôpital Hautepierre; L. Mouthon, MD, PhD, Professor, Paris Descartes Université, Médecine Interne, Hôpital Cochin, APHP, J. Cabane, MD, PhD, Professor, Université Pierre et Marie Curie, Service de Médecine Interne, Hôpital Saint-Antoine, APHP; Z. Amoura, $M D, P h D$, Professor, Université Paris 6, Service de Médecine Interne 2, Hôpital Pitié-Salpêtrière, AP-HP; J-L. Crakowski, MD, PhD, Laboratoire de Pharmacologie, INSERM ESPRI, HP2 EA 3745, Faculté de Médecine de Grenoble; P. Carpentier, MD, PhD, Clinique Universitaire De Médecine Vasculaire, Pôle Pluridisciplinaire de Médecine, Centre Hospitalier Universitaire; J. Avouac, MD, PhD, INSERM U781, Université Paris Descartes, Hôpital Necker, Rhumatologie A, Hôpital Cochin, APHP; O. Meyer, MD, Professor, Université Diderot-Paris 7 , Service de Rhumatologie, Hôpital Bichat Claude Bernard, APHP; A. Kahan, MD, PhD, Professor, Université Paris Descartes, Rhumatologie A, Hôpital Cochin, APHP; C. Boileau, PharmD, PhD, Professor, INSERM U781, Université Paris Descartes, Hôpital Necker, Laboratoire de Biochimie Hormonale et Génétique, Hôpital Ambroise Paré, AP-HP, Université Versailles Saint Quentin Yvelines; Y. Allanore, MD, PhD, Prof, INSERM U781, Université Paris Descartes, Hôpital Necker, Rhumatologie A, Hôpital Cochin, APHP.

Address correspondence to Dr. P. Dieudé, Service de Rhumatologie, Hôpital Bichat Claude Bernard, 46 rue Henri Huchard, 75018 Paris, France.E-mail: philippe.dieude@bch.aphp.fr Accepted for publication December 29, 2009. 
Systemic sclerosis ( $\mathrm{SSc}$ ) is an orphan complex multiorgan disease affecting the microvascular network, the immune system, and connective tissue. Progressive organ failure makes SSc a severe chronic disease leading to major disability or death ${ }^{1}$. To date no drug has proven its efficacy to counteract the generalized multiorgan fibrotic process ${ }^{2}$. Recent data have highlighted the poor efficacy of current available drugs, in particular in the 2 major causes of death, interstitial lung disease ${ }^{3}$ and pulmonary arterial hypertension ${ }^{4}$.

The type 1 interferon (IFN) pathway has been postulated to play a key role in autoimmune diseases ${ }^{5}$. Indeed, an increased expression of type 1 IFN genes by peripheral blood mononuclear cells, referred to as an IFN signature, has been detected in multiple autoimmune diseases such as systemic lupus erythematosus (SLE), rheumatoid arthritis (RA), and Sjögren's syndrome (SS) and in a subgroup of patients with $\mathrm{SSc}^{6-9}$. The interferon regulatory factors (IRF) are major regulators of genes activated by the type 1 IFN. Recently, the interferon regulatory factor 5 gene (IRF5) has been identified as an autoimmune disease susceptibility gene in the background of SS, SLE, RA, and $\mathrm{SSc}^{10-13}$. Our original finding of an association between the IRF5 and SSc provided additional support for the pivotal role of IRF5 and the type 1 IFN pathway in $\mathrm{SSc}^{14}$. We found that the functional IRF5 rs2004640 single-nucleotide polymorphism (SNP), which creates a donor splice site in intron 1 of the IRF5 gene resulting in transcription of the alternative exon 1B, displayed association with SSc in 2 independent patient cohorts from French Caucasian populations ${ }^{14}$. Further, our data provided new clues for a role of IRF5 and IFN pathways in fibrosing alveolitis. Recently, different additional functional IRF5 variants have been convincingly identified as autoimmune genetic factors. Among these IRF5 variants, the rs 10954213 SNP alters the polyadenylation site of IRF5, the rare $\mathrm{A}$ allele leading to a shorter and more stable mRNA $^{15}$. A 5-bp biallelic insertion deletion polymorphism (CGGGG indel) located in the first intron of the IRF5 gene, resulting in increased binding of the transcription factor SP1 to the risk allele of the 5-bp indel, was also reported to confer susceptibility to autoimmunity ${ }^{16-18}$. Interestingly, the functional CGGGG indel polymorphism could be unambiguously inferred from haplotype reconstructions due to the high linkage disequilibrium with the 3 following SNP: rs 377385 , rs2004640, and rs $10954213^{17}$. Therefore, the aim of our study was to investigate whether IRF5 haplotypes determined by those 3 SNP may be also involved in the genetic background of SSc and to account for the respective subphenotypes.

\section{MATERIALS AND METHODS}

Study population and design. We performed a large case-control association study including 827 SSc patients and 989 controls from the French SSc network as described ${ }^{14,19}$. All SSc patients were classified according to the cutaneous subtype classification of LeRoy, et $a l^{20}$, and phenotypically assessed as recommended ${ }^{21}$. Controls consisted of healthy unrelated indi- viduals ethnically matched to the SSc cases. All subjects were of white French Caucasian ethnicity, defined by all 4 grandparents being white French Caucasian. The ethics committee of Cochin Hospital, Paris, approved the study and all subjects gave their written informed consent.

As reported, SSc cases with co-occurrence of a known IRF5-associated autoimmune disease (SLE and/or SS and/or RA and/or multiple sclerosis) were excluded prior to the analyses ${ }^{14}$. As reported, SSc patients with any ground-glass opacity on thoracic high-resolution computed tomography scan were considered to have interstitial lung involvement ${ }^{22,23}$. Pulmonary vascular and heart involvements were assessed by cardiac echocardiography and right-heart catheterization in cases suspected of having pulmonary arterial hypertension ${ }^{24}$.

Autoantibody analysis. SSc patients were tested for antinuclear antibodies (ANA) using indirect immunofluorescence (IIF) and HEp-2 cells as antigen substrate (Antibodies Inc., Davis, CA, USA). Specific SSc antibodies were systematically assessed; anticentromere antibodies (ACA) were determined by their distinctive IIF pattern on HEp-2 cells. Anti-topoisomerase I antibodies were determined by counter-immunoelectrophoresis.

Genotyping. The 3 SNP were genotyped using a competitive allele-specific polymerase chain reaction (PCR) system (Kaspar genotyping, Kbioscience, Hoddeston, UK) and the Taqman SNP genotyping assay-allelic discrimination method (Applied Biosystems, Foster City, CA, USA) as described $^{14,19}$. IRF5 rs2004640 was previously genotyped for the same individuals in our cohort ${ }^{25}$. The average genotype completeness for the 3 SNP tested was $89 \%$ for the SSc and control samples. The accuracy was > $99 \%$, by duplicate genotyping of $10 \%$ of all samples. The CGGGG indel polymorphic marker was amplified by fluorescent PCR and genotyped on an ABI-Prism 3100 device (Applied Biosystems, Courtaboeuf, France) and analyzed with Genotyper software in 100 (i.e., 200 chromosomes) randomly selected individuals homozygous for IRF5 risk haplotype and in 40 (80 chromosomes) individuals homozygous for the protective haplotype.

Statistical analysis. Single-marker and haplotype analyses were performed in the SSc patients and controls having full genotype information for the 3 IRF5 SNP (743 SSc patients, 880 controls). Statistical analyses were performed using the R software (version 2.6.0). The level of significance for all tests corresponds to a type I error rate $\alpha=5 \%$. The Bonferroni correction was applied for all the tests for a "hypothesis-generating step" comparing SSc cases and controls (10 phenotypic subsets; $\mathrm{p}$ values adjusted). All odds ratios are provided with $95 \%$ confidence interval. Conformity to Hardy-Weinberg equilibrium was tested using a classical 1 degree of freedom chi-square test in controls. Individual association analyses of IRF5 SNP with SSc were performed by comparing cases and controls with Fisher's exact test on genotypes. Corresponding odds ratios were assessed using standard logistic regression analysis with the most frequent genotype taken as the reference. The same procedure was applied in subgroups stratified according to SSc phenotypes. An analysis of haplotype diversity was realized for the 3 SNP with the expectation-maximization (EM) algorithm as implemented in the haplo.stats R library. Specific p values and odds ratios were obtained by comparing each haplotype with the more common haplotype in the population, applying Fisher's exact test.

\section{RESULTS}

IRF5 single-marker analysis. Genotype frequencies of the 3 IRF5 SNP were in Hardy-Weinberg equilibrium in the control population.

Among the 829 patients and 989 controls investigated, complete genotyping data for the 3 IRF5 variants were available for 743 and 880 individuals, respectively. Allelic and genotypic frequencies of the IRF5 rs3757385, rs2004640, and rs10954213 SNP were in good agreement with frequencies reported among the European Caucasian population ${ }^{17}$. The case-control association study involving 
743 SSc cases and 880 controls revealed an association between IRF5 rs3757385, rs2004640, and rs 10954213 variants and SSc (Table 1). The magnitude of the association was most important for IRF5 homozygous genotypes for the risk allele of rs3757385 and rs2004640 SNP, suggesting a dosage effect ( $\mathrm{p}=0.009$, OR $1.51,95 \%$ CI $1.10-2.08$ and $\mathrm{p}=3.5 \times 10^{-5}$, OR $1.81,95 \%$ CI 1.36-2.40, respectively).

We tested whether the associated SNP constituted an independent effect. Pair-wise correlation coefficient $\left(\mathrm{r}^{2}\right)$ analysis found rs3757385 in relatively high linkage disequilibrium with $r$ s2004640 $\left(r^{2}=0.57\right)$ and $r s 10954213\left(r^{2}=0.56\right)$. Conversely, rs2004640 was not in strong linkage disequilibrium with $\mathrm{rs} 10954213\left(\mathrm{r}^{2}=0.34\right)$. Multiple logistic regression analysis of the 3 SNP revealed no independent effect: the rs2004640 was the only variant that remained significantly associated conditionally to the 2 others $(\mathrm{p}=0.0086)$.

IRF5 haplotype analysis. Among the 8 IRF5 haplotypes defined by the 3 IRF5 rs3757385, rs2004640, and rs 10954213 SNP, 4 common haplotypes (frequency $>5 \%$ in both controls and cases) were predicted from our sample. Of the 4 haplotypes, 2 were at a frequency of $25 \%$ or more in the controls. As previously observed in a European Caucasian population ${ }^{17}$, these 2 common haplotypes C-T-A and A-G-G were present at a frequency of $44 \%$ and $33 \%$, respectively, in our control chromosomes. Taking into account the IRF5 risk alleles $\mathrm{rs} 3757385^{*} \mathrm{C}$, rs $2004640 * \mathrm{~T}$, and rs $10954213^{*} \mathrm{~A}$, we tested for association of the IRF5 C-T-A risk haplotype ("R" haplotype). The IRF5 "R" haplotype was associated with SSc as its frequency was significantly increased in SSc patients (49\%) compared to controls
$(44 \%)\left(\mathrm{p}_{\text {adj }}=0.024\right.$, OR $1.23,95 \%$ CI $\left.1.07-1.40\right)$. We also observed that the C-T-A "mirror" haplotype, i.e., the A-G-G haplotype, acts with a protective ("P" haplotype) effect, its frequency being decreased in the SSc population (27.8\%) compared to controls $(32.9 \%)\left(\mathrm{p}_{\mathrm{adj}}=8.8 \times 10^{-3}\right.$, OR 0.78 , 95\% CI 0.68-0.90; Table 2).

Phenotype-genotype correlation in SSc. We previously reported results identifying IRF5 rs2004640 as a new SSc susceptibility gene, with a strong effect in the genetic background of diffuse cutaneous SSc (dcSSc) and SSc-related fibrosing alveolitis ${ }^{14}$. Following those results we investigated the effect of the IRF5 "R" and "P" haplotypes in subsets of SSc [dcSSc, limited cutaneous SSc (lcSSc), and patients with and without fibrosing alveolitis].

In this study, we observed a strong association of the IRF5 rs2004640 TT genotype with both dcSSc ( $\mathrm{p}_{\text {adj }}=2.6 \times$ $10^{-3}$, OR 2.01, 95\% CI 1.39-3.05) and SSc-related pulmonary fibrosis $\left(\mathrm{p}_{\mathrm{adj}}=3.7 \times 10^{-4}\right.$, OR $2.19,95 \% \mathrm{CI}$ $1.50-3.20$ ). The TT risk genotype was also strongly associated with the subset of severe organ involvement, compared to controls ( $\mathrm{p}_{\mathrm{adj}}=2.2 \times 10^{-4}$, OR 2.16, 95\% CI 1.50-3.09). The IRF5 rs3757385 variant was also found to be associated with the different SSc subsets; conversely, no association was detected with rs 10954213 (Table 1).

However, regarding the phenotype-genotype correlation, intra-cohort analyses failed to detect any significant differences comparing the allele and genotype frequencies of the 3 IRF5 SNP and the 3 subsets (i.e., cutaneous phenotype, pulmonary fibrosis, and severe organ involvement; data not shown).

Table 1. Genotype and allele frequency comparisons of the IRF5 rs3757385, rs2004640, and rs10954213 polymorphisms in SSc patients and controls. For each IRF5 SNP p values are given as follows: risk allele, homozygous and heterozygous genotypes for the risk allele.

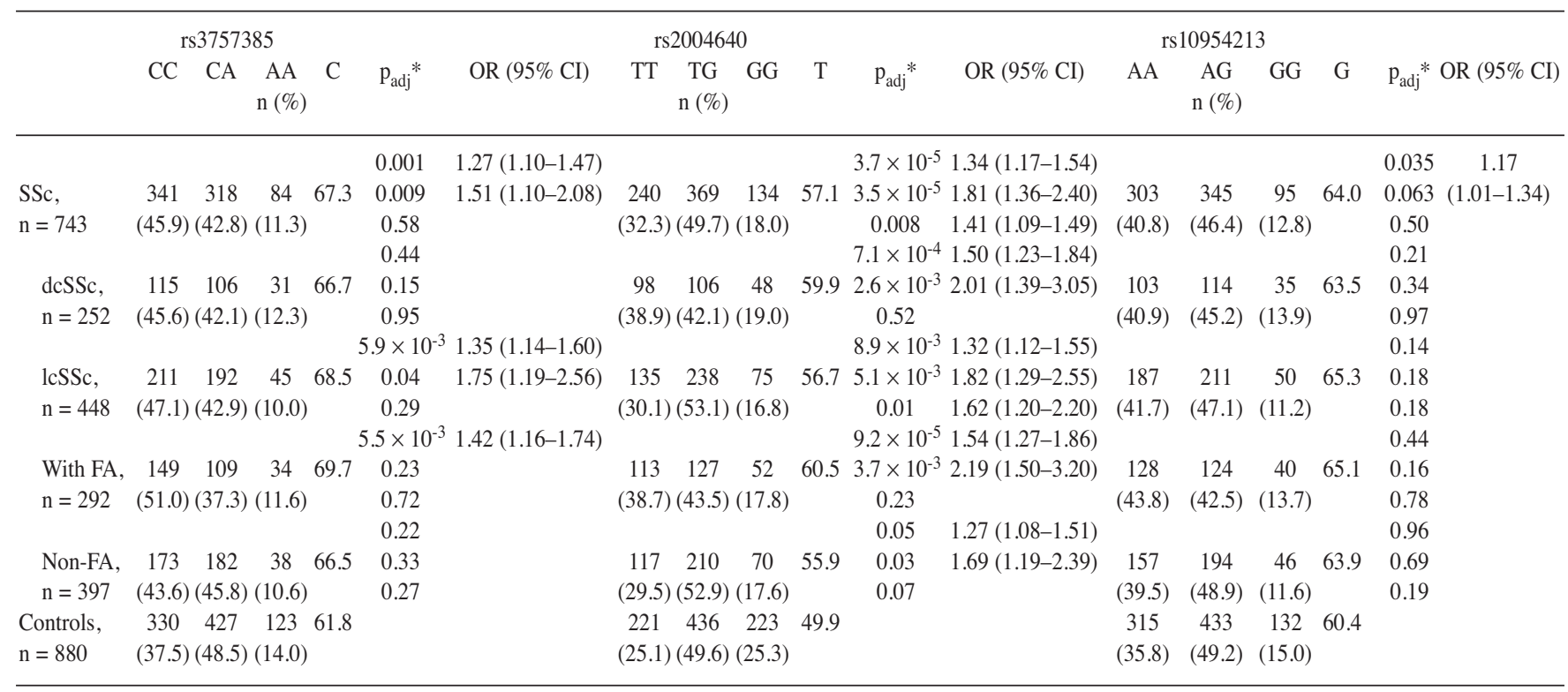

* Adjusted after Bonferroni correction for multiple comparisons. dcSSc: diffuse cutaneous systemic sclerosis; lcSSc: limited cutaneous systemic sclerosis; FA: fibrosing alveolitis. 
Table 2. IRF5 haplotype distribution in SSc patients and controls and haplotype-phenotype correlation.

\begin{tabular}{|c|c|c|c|c|}
\hline & $\begin{array}{l}\text { IRF5 Haplotype } \\
\text { Frequency, \% }\end{array}$ & $\mathrm{p}_{\mathrm{adj}}{ }^{*}$ & $\mathrm{p}^{* *}$ & OR $(95 \% \mathrm{CI})$ \\
\hline \multicolumn{5}{|c|}{ IRF5 C-T-A risk haplotype (R) } \\
\hline $\mathrm{SSc}, \mathrm{n}=743$ & 48.9 & 0.024 & \multirow{4}{*}{0.0081} & $1.23(1.07-1.40)$ \\
\hline $\mathrm{dcSSc}, \mathrm{n}=252$ & 50.9 & 0.037 & & $1.32(1.10-1.59)$ \\
\hline & & & & \\
\hline $1 \mathrm{cSSc}, \mathrm{n}=448$ & 43.8 & NS & & \\
\hline \multirow[t]{2}{*}{$\mathrm{FA}, \mathrm{n}=292$} & 50.6 & 0.037 & \multirow{4}{*}{ NS } & $1.32(1.09-1.59)$ \\
\hline & & & & \\
\hline Non-FA, $n=397$ & 48.4 & NS & & \\
\hline Controls, $\mathrm{n}=880$ & 43.8 & & & \\
\hline \multicolumn{5}{|c|}{ IRF5 A-G-G protective haplotype (p) } \\
\hline $\mathrm{SSc}, \mathrm{n}=743$ & 27.8 & $8.8 \times 10^{-3}$ & \multirow{4}{*}{ NS } & $0.78(0.68-0.90)$ \\
\hline \multirow[t]{2}{*}{$\mathrm{dcSSc}, \mathrm{n}=252$} & 28.2 & NS & & \\
\hline & & & & \\
\hline lcSSc, $n=448$ & 32.9 & NS & & \\
\hline \multirow[t]{2}{*}{$\mathrm{FA}, \mathrm{n}=292$} & 24.1 & $3.7 \times 10^{-4}$ & \multirow{4}{*}{0.018} & $0.64(0.51-0.79)$ \\
\hline & & & & \\
\hline Non-FA, $\mathrm{n}=397$ & 29.6 & NS & & \\
\hline Controls, $\mathrm{n}=880$ & 32.9 & & & \\
\hline
\end{tabular}

\footnotetext{
* Adjusted for comparison between SSc patients and controls. ** Comparison between SSc patients with and without a specific phenotype (i.e., dcSSc vs lcSSc, SSc patients with FA vs those without, severe subset vs non-severe subset). NS: not statistically significant; other abbreviations as in Table 1.
}

Phenotype-haplotype correlation in SSc. Regarding the IRF5 C-T-A "R" haplotype, we found an association restricted to dcSSc $\left(\mathrm{p}_{\mathrm{adj}}=0.037\right.$, OR $1.32,95 \%$ CI $1.10-1.59)$. The IRF5 A-G-G "P" haplotype was found to act with a protective effect restricted to the diffuse cutaneous subtype and the subset of SSc patients with fibrosing alveolitis (Table 2). When phenotype-haplotype correlation was investigated, intra-cohort analyses revealed a significantly increased frequency of the "R" haplotype in dcSSc compared to lcSSc $(\mathrm{p}=0.0081)$. Conversely, the "P" haplotype was found to be underrepresented in the subset of SSc patients having fibrosing alveolitis compared to those without ( $\mathrm{p}=0.018)$; the frequency of the " $\mathrm{R}$ " haplotype did not differ between the 2 SSc subsets (Table 2). As fibrosing alveolitis is reported to be linked to dcSSc, we next compared the frequencies of the IRF5 "P" haplotype between patients with and those without fibrosing alveolitis in the lcSSc subset: we observed a decreased frequency of the "P" haplotype in the patient subset without fibrosing alveolitis; however, this did not achieve statistical significance (data available upon request from the author).

As previously stated, the susceptibility CGGGG indel allele was inferred from the 2 IRF5 "R" and "P" haplotypes. Among a group of $100 \mathrm{SSc}$ patients and controls who were homozygous for the "R" haplotype, $100 \%$ were carrying the CGGGG insertion allele; conversely, among 40 individuals homozygous for the IRF5 "P" haplotype, none had the CGGGG indel susceptibility allele, corroborating that the "R" haplotype harbors the CGGGG indel risk allele.

\section{DISCUSSION}

We previously reported an association between the functional IRF5 rs2004640 polymorphism and $\mathrm{SSc}^{14}$. Identification of IRF5 as a genetic susceptibility factor shared by various autoimmune diseases is an important step for understanding type I IFN-driven autoimmunity. Further, this has generated new clues about interstitial lung disease in the context of SSc, which has become a leading cause of death, without established therapeutic options. However, to date, many IRF5 functional variants have been identified as risk factors for different autoimmune diseases and remain to be investigated in SSc. Our study is the first to provide evidence for association between 2 IRF5 haplotypes and SSc. We identify an IRF5 risk haplotype ("R") defined by the alleles rs $3757385^{*} \mathrm{C}$, rs2004640*T, and rs $10954213^{*} \mathrm{~A}$ (C-T-A) that confers susceptibility to SSc. The IRF5 "R" haplotype was found to confer susceptibility to defined subsets of SSc including dcSSc and SSc-related pulmonary fibrosis. These findings illustrate that IRF5 may contribute to the typical heterogeneity of SSc. This observation is in good agreement with our original study ${ }^{14}$, as the main subphenotype associations were the diffuse cutaneous and fibrosing alveolitis subsets. In this sample, intra-cohort single-marker analyses failed to provide evidence for using one of the 3 IRF 5 variants as a marker for a particular SSc subset. This discrepancy with our previous findings could be related to the cross-sectional design of the study and a smaller sample size ( $743 \mathrm{SSc}$ cases, 880 controls) leading to a weaker power to detect such intra-cohort associations. Of 
interest, phenotype-haplotype correlation analysis did detect intra-cohort association signals. Indeed, IRF5 " $\mathrm{R}$ " and/or "P" haplotypes were able to discriminate dcSSc and fibrosing alveolitis. Those results emphasize that IRF5 haplotype analyses are more informative than single-marker analyses of rs3757385, rs2004640, and rs10954213, suggesting the haplotype biomarker could be helpful for risk stratification of patients with Ssc.

It is noteworthy that the same risk haplotype has been reported to be associated with high serum levels of type 1 IFN in patients with $\mathrm{SLE}^{26}$. It could be hypothesized that the IRF5 "R" haplotype harbors one or multiple causal polymorphisms. Interestingly, a 5-bp biallelic insertion-deletion polymorphism (CGGGG indel) located in the first intron of the IRF5 gene was recently reported to be associated with multiple autoimmune diseases ${ }^{16-18}$. The CGGGG risk insertion, which results in increased binding of the SP1 transcription factor, has recently been reported to regulate the expression of IRF5 mRNA ${ }^{27,28}$. The CGGGG risk insertion suggested to be the causal variant of IRF5 in SLE and in primary SS could be unambiguously inferred from haplotype reconstructions with the 3 IRF5 SNP tested in our study ${ }^{17}$. Hence, the IRF5 " $\mathrm{R}$ " haplotype is the exclusive haplotype carrying the CGGGG insertion risk allele, suggesting a possible contribution of this functional variant in the genetic background of $\mathrm{SSc}^{17}$.

As previously reported, the susceptibility CGGGG indel allele was unambigously inferred from the 2 IRF5 " $R$ " and "P" haplotypes in a French Caucasian population. This haplotype was also reported to confer susceptibility to multiple sclerosis in the European Caucasian population ${ }^{17}$. However, resequencing of the IRF5 gene in patients homozygous for the " $R$ " haplotype is required for a conclusion that the CGGGG insertion deletion is the definite IRF5 causal variant. Further, it is notable that multiple IRF5 SNP including rs2070197 and rs12539741, located in the 3'UTR, were reported to confer susceptibility to SLE and may be involved in SSc susceptibility ${ }^{15}$. Of interest, those SNP are in perfect linkage disequilibrium with several additional Hapmap SNP distributed over a 10-kb region located 3' of the IRF5 gene that contains the transportin 3 (TNPO3) gene. The reported pair-wise linkage disequilibrium between these SNP and the CGGGG indel is very low, suggesting that the SNP could tag an independent SLE signal association; this remains to be investigated in $\mathrm{SSc}^{16}$.

Genetic studies of SSc susceptibility have made great progress, and considering the current efforts, more genetic risk factors will be identified. In contrast, the field of the genetics of SSc heterogeneity is relatively unexplored. Following our results, IRF5 should be investigated in genetics of SSc phenotypic heterogeneity. Identification of the IRF5 gene as a susceptibility factor for multiple autoimmune disorders emphasizes that similar immunogenetic mechanisms underlie autoimmune diseases. It is also impor- tant for understanding type I IFN-driven autoimmunity IFN regulatory factors (IRF), such as IRF5, coordinate the expression of type I IFN, which are assumed to participate in the pathogenesis of connective tissue disorders including SLE, SS, and SSc ${ }^{6,7,9}$. IRF5 also coordinates the expression of interleukin $6^{29}$, which was found to be elevated in plasma serum of patients with dcSSc and SSc-related pulmonary fibrosis $^{30,31}$. Increased understanding of the role of genetics in disease outcomes in SSc may promote the development of new therapies.

Our study provides evidence for IRF5 as a new SSc gene playing a key role in disease severity, and supports the pivotal role of type 1 IFN in the pathophysiology of SSc. Further research is needed on the pathogenesis of this severe autoimmune disease, for which there is no cure.

\section{ACKNOWLEDGMENT}

The authors thank Dr. Joëlle Benessiano, Centre de Ressource Biologique, Hôpital Bichat, Paris, France; Établissement Français du Sang (EFS); and Prof. Bernard Granchamp and Dr. Nadem Soufir for their assistance in setting up the control samples.

\section{REFERENCES}

1. Allanore Y, Avouac J, Kahan A. Systemic sclerosis: an update in 2008. Joint Bone Spine 2008;75:650-5.

2. Allanore Y, Wipff J, Kahan A, Boileau C. Genetic basis for systemic sclerosis. Joint Bone Spine 2007;74:577-83.

3. Steen VD, Medsger TA. Changes in causes of death in systemic sclerosis, 1972-2002. Ann Rheum Dis 2007;66:940-4

4. Condliffe R, Kiely DG, Peacock AJ, Corris PA, Gibbs JS, Vrapi F, et al. Connective tissue disease-associated pulmonary arterial hypertension in the modern treatment era. Am J Respir Crit Care Med 2009; 179:151-7

5. Ronnblom LE, Alm GV, Oberg KE. Autoimmunity after alpha-interferon therapy for malignant carcinoid tumors. Ann Intern Med 1991;115:178-83.

6. Baechler EC, Batliwalla FM, Karypis G, Gaffney PM, Ortmann WA, Espe KJ, et al. Interferon-inducible gene expression signature in peripheral blood cells of patients with severe lupus. Proc Natl Acad Sci USA 2003;100:2610-5.

7. Gottenberg JE, Cagnard N, Lucchesi C, Letourneur F, Mistou S, Lazure T, et al. Activation of IFN pathways and plasmacytoid dendritic cell recruitment in target organs of primary Sjogren's syndrome. Proc Natl Acad Sci USA 2006;103:2770-5.

8. Baechler EC, Batliwalla FM, Reed AM, Peterson EJ, Gaffney PM, Moser KL, et al. Gene expression profiling in human autoimmunity. Immunol Rev 2006;210:120-37.

9. Tan FK, Zhou X, Mayes MD, Gourh P, Guo X, Marcum C, et al. Signatures of differentially regulated interferon gene expression and vasculotrophism in the peripheral blood cells of systemic sclerosis patients. Rheumatology 2006;45:694-702.

10. Graham RR, Kozyrev SV, Baechler EC, Reddy MV, Plenge RM, Bauer JW, et al. A common haplotype of interferon regulatory factor 5 (IRF5) regulates splicing and expression and is associated with increased risk of systemic lupus erythematosus. Nat Genet 2006;38:550-5.

11. Sigurdsson S, Nordmark G, Goring HH, Lindroos K, Wiman AC, Sturfelt $\mathrm{G}$, et al. Polymorphisms in the tyrosine kinase 2 and interferon regulatory factor 5 genes are associated with systemic lupus erythematosus. Am J Hum Genet 2005;76:528-37.

12. Miceli-Richard C, Comets E, Loiseau P, Puechal X, Hachulla E, 
Mariette X. Association of an IRF5 gene functional polymorphism with Sjogren's syndrome. Arthritis Rheum 2007;56:3989-94.

13. Han SW, Lee WK, Kwon KT, Lee BK, Nam EJ, Kim GW. Association of polymorphisms in interferon regulatory factor 5 gene with rheumatoid arthritis: a metaanalysis. J Rheumatol 2009;36:693-7.

14. Dieude P, Guedj M, Wipff J, Avouac J, Fajardy I, Diot E, et al. Association between the IRF5 rs2004640 functional polymorphism and systemic sclerosis: a new perspective for pulmonary fibrosis. Arthritis Rheum 2009;60:225-33.

15. Graham RR, Kyogoku C, Sigurdsson S, Vlasova IA, Davies LR, Baechler EC, et al. Three functional variants of IFN regulatory factor 5 (IRF5) define risk and protective haplotypes for human lupus. Proc Natl Acad Sci USA 2007;104:6758-63.

16. Dideberg V, Kristjansdottir G, Milani L, Libioulle C, Sigurdsson S, Louis E, et al. An insertion-deletion polymorphism in the interferon regulatory factor 5 (IRF5) gene confers risk of inflammatory bowel diseases. Hum Mol Genet 2007;16:3008-16.

17. Kristjansdottir G, Sandling JK, Bonetti A, Roos IM, Milani L, Wang $\mathrm{C}$, et al. Interferon regulatory factor 5 (IRF5) gene variants are associated with multiple sclerosis in three distinct populations. J Med Genet 2008;45:362-9.

18. Nordmark G, Kristjansdottir G, Theander E, Eriksson P, Brun JG, Wang C, et al. Additive effects of the major risk alleles of IRF5 and STAT4 in primary Sjogren's syndrome. Genes Immun 2009;10:68-76.

19. Dieude P, Guedj M, Wipff J, Avouac J, Hachulla E, Diot E, et al. The PTPN22 620W allele confers susceptibility to systemic sclerosis: findings of a large case-control study of European Caucasians and a meta-analysis. Arthritis Rheum 2008;58:2183-8.

20. LeRoy EC, Black C, Fleischmajer R, Jablonska S, Krieg T, Medsger TA Jr, et al. Scleroderma (systemic sclerosis): classification, subsets and pathogenesis. J Rheumatol 1988; 15:202-5.

21. Valentini G, Matucci Cerinic M. Disease-specific quality indicators, guidelines and outcome measures in scleroderma. Clin Exp Rheumatol 2007;25 Suppl:159-62.

22. Tashkin DP, Elashoff R, Clements PJ, Goldin J, Roth MD, Furst DE, et al. Cyclophosphamide versus placebo in scleroderma lung disease. N Engl J Med 2006;354:2655-66.
23. Gilson M, Zerkak D, Wipff J, Dusser D, Dinh-Xuan AT, Abitbol V, et al. Prognostic factors for lung function in systemic sclerosis: prospective study of 105 cases. Eur Respir J 2010;35:112-7.

24. Allanore Y, Borderie D, Avouac J, Zerkak D, Meune C, Hachulla E, et al. High $\mathrm{N}$-terminal pro-brain natriuretic peptide levels and low diffusing capacity for carbon monoxide as independent predictors of the occurrence of precapillary pulmonary arterial hypertension in patients with systemic sclerosis. Arthritis Rheum 2008;58:284-91.

25. Dieude P, Guedj M, Wipff J, Ruiz B, Hachulla E, Diot E, et al. STAT4 is a genetic factor for systemic sclerosis having additive effects with IRF5 on disease susceptibility and related pulmonary fibrosis. Arthritis Rheum 2009;60:2472-9.

26. Niewold TB, Kelly JA, Flesch MH, Espinoza LR, Harley JB, Crow MK. Association of the IRF5 risk haplotype with high serum interferon-alpha activity in systemic lupus erythematosus patients. Arthritis Rheum 2008;58:2481-7.

27. Sigurdsson S, Goring HH, Kristjansdottir G, Milani L, Nordmark $\mathrm{G}$, Sandling JK, et al. Comprehensive evaluation of the genetic variants of interferon regulatory factor 5 (IRF5) reveals a novel 5 bp length polymorphism as strong risk factor for systemic lupus erythematosus. Hum Mol Genet 2008;17:872-81.

28. Miceli-Richard C, Gestermann N, Ittah M, Comets E, Loiseau P, Puechal X, et al. The CGGGG insertion/deletion polymorphism of the IRF5 promoter is a strong risk factor for primary Sjogren's syndrome. Arthritis Rheum 2009;60:1991-7.

29. Takaoka A, Yanai H, Kondo S, Duncan G, Negishi H, Mizutani T, et al. Integral role of IRF-5 in the gene induction programme activated by Toll-like receptors. Nature 2005;434:243-9.

30. Hasegawa M, Sato S, Fujimoto M, Ihn H, Kikuchi K, Takehara K. Serum levels of interleukin 6 (IL-6), oncostatin M, soluble IL-6 receptor, and soluble gp130 in patients with systemic sclerosis. J Rheumatol 1998;25:308-13.

31. Gourh P, Arnett FC, Assassi S, Tan FK, Huang M, Diekman L, et al. Plasma cytokine profiles in systemic sclerosis: associations with autoantibody subsets and clinical manifestations. Arthritis Res Ther 2009;11:R147. 